\title{
A Sample Preparation Pipeline for Microcrystals at the VMXm Beamline
}

\author{
Adam D. Crawshaw ${ }^{1}$, Emma V. Beale ${ }^{1,2}$, Anna J. Warren ${ }^{1}$, Andrew Stallwood ${ }^{1,3}$, Graham Duller ${ }^{1}$, Jose Trincao ${ }^{1}$, Gwyndaf \\ Evans $^{1,4}$ \\ ${ }^{1}$ Diamond Light Source Ltd, Harwell Science and Innovation Campus ${ }^{2}$ Paul Scherrer Institut ${ }^{3}$ Central Laser Facility, Science and Technologies Facilities \\ Council, Harwell Science and Innovation Campus ${ }^{4}$ Rosalind Franklin Institute, Harwell Science and Innovation Campus
}

\section{Corresponding Author}

Adam D. Crawshaw

adam.crawshaw@diamond.ac.uk

\section{Citation}

Crawshaw, A.D., Beale, E.V.,

Warren, A.J., Stallwood, A., Duller, G.,

Trincao, J., Evans, G. A Sample

Preparation Pipeline for Microcrystals at the VMXm Beamline. J. Vis. Exp. (172), e62306, doi:10.3791/62306 (2021).

\section{Date Published}

June 17, 2021

DOI

$10.3791 / 62306$

URL

jove.com/video/62306

\section{Abstract}

The mounting of microcrystals $(<10 \mu \mathrm{m})$ for single crystal cryo-crystallography presents a non-trivial challenge. Improvements in data quality have been seen for microcrystals with the development of beamline optics, beam stability and variable beam size focusing from submicron to microns, such as at the VMXm beamline at Diamond Light Source ${ }^{1}$. Further improvements in data quality will be gained through improvements in sample environment and sample preparation. Microcrystals inherently generate weaker diffraction, therefore improving the signal-to-noise is key to collecting quality $\mathrm{X}$-ray diffraction data and will predominantly come from reductions in background noise. Major sources of X-ray background noise in a diffraction experiment are from their interaction with the air path before and after the sample, excess crystallization solution surrounding the sample, the presence of crystalline ice and scatter from any other beamline instrumentation or X-ray windows. The VMXm beamline comprises instrumentation and a sample preparation protocol to reduce all these sources of noise.

Firstly, an in-vacuum sample environment at VMXm removes the air path between X-ray source and sample. Next, sample preparation protocols for macromolecular crystallography at VMXm utilize a number of processes and tools adapted from cryoTEM. These include copper grids with holey carbon support films, automated blotting and plunge cooling robotics making use of liquid ethane. These tools enable the preparation of hundreds of microcrystals on a single cryoTEM grid with minimal surrounding liquid on a low-noise support. They also minimize the formation of crystalline ice from any remaining liquid surrounding the crystals.

We present the process for preparing and assessing the quality of soluble protein microcrystals using visible light and scanning electron microscopy before mounting the samples on the VMXm beamline for X-ray diffraction experiments. We will also provide 


\section{Introduction}

A major barrier for the determination of high-resolution structures of biological molecules by macromolecular crystallography (MX) remains the production of well diffracting crystals at an amenable size. There are many strategies for achieving this goal from recombinant protein gene construct design through to large sparse matrix searches for chemical cocktails that may generate initial crystals ${ }^{2}$. For the latter, it is often the case that the crystallographer will need to optimize any initial hits to obtain crystals with sufficient diffraction quality and size for structure determination studies $^{3}$. Despite these options, some target molecules may never generate large $(>10 \mu \mathrm{m})$, well diffracting crystals and as a result the crystallographer must persevere with their microcrystals and the challenges that such samples present. These include appropriately mounting and cryo-protecting the crystals, managing inherently weaker diffraction and increased radiation sensitivity. Microcrystals are formed from fewer unit cells and molecules than larger crystals and as such, the diffraction is not amplified to the same extent compared to larger crystals, resulting in inherently weaker diffraction intensities. It is important that the background signal does not mask these reflections, particularly at higher resolution where weak reflection intensities can be lost ${ }^{4}$. In addition, microcrystals are more sensitive to radiation damage and despite recording diffraction at liquid nitrogen temperatures ${ }^{5}$, it may not be possible to collect complete data from a single crystal, making it necessary to collect data from a very large number of crystals to produce a single complete dataset $^{6}$.

The increasing availability of $\mathrm{X}$-ray free electron lasers (XFELs) and the evolution of serial crystallography methods $(\mathrm{SFX})^{7}$ have provided routes to collecting data from smaller microcrystals. However, these are bespoke sample delivery methods, which require a significant amount of hardware and software expertise, where experiments are limited to room-temperature and typically sample consumption is high (hundreds of microliters) and still may require further optimisation ${ }^{8}$. As such, projects where only a limited quantity of microcrystals can be made are not appropriate for SFX.

Meanwhile, synchrotron beamline technology over recent decades has progressed to produce smaller, more stable beams ${ }^{9}$ with a brilliance that has permitted data collection from ever smaller crystals ${ }^{10,11}$. Microfocus beamlines such as FMX at NSLS-II and I24 at Diamond Light Source have been able to determine novel structures from crystals with maximum dimensions of $\sim 3 \mu \mathrm{m}^{12}$ and demonstrate the ability to collect usable data from even smaller crystals measuring $\sim 1 \mu \mathrm{m}^{13}$. The beamline must be precisely configured, with excellent, high resolution on-axis-viewing optics, a minimal sphere of confusion for sample rotation and a precisely aligned rotation axis that is coincident with the $\mathrm{X}$-ray beam. It is important to closely match the X-ray beam profile to the crystal volume and ensure the crystal is precisely aligned in the X-ray beam - a challenge for crystals $<5 \mu \mathrm{m}^{14}$. Meeting 
these experimental conditions at the beamline is essential to recording the best quality data from microcrystals.

The remaining and possibly most important aspect of data collection from microcrystals is the presentation of the crystal to the X-ray beam. Microcrystals have often been mounted on micromesh sample mounts, manufactured from polyimide, a low X-ray scattering material with apertures as small as 10 $\mu \mathrm{m}^{15,16}$. The polyimide mesh is mounted on a standard pin that is set into a magnetic SPINE base, making it compatible with most $\mathrm{MX}$ beamlines ${ }^{17}$. The mesh mount is used to fish crystals from the crystallization drop often following the same procedure as mounting a $100 \mu \mathrm{m}$ crystal using a standard loop style mount. While the crystals may be distributed across the mesh, a key disadvantage is that a relatively large volume of liquid can be carried by the mesh and the pin while harvesting (Figure 1C,D). This volume of liquid, that can be many times larger than the crystals themselves, will contribute to background noise when illuminated with $\mathrm{X}$ rays. This background scatter can be even stronger if the liquid forms crystalline ice during flash cooling, diminishing the signal-to-noise ratio of already weak intensities within the resolutions of ice diffraction. Therefore, it is key that excess liquid is removed from the sample, to ensure that all possible signals can be recorded. This challenge is even greater in the case of membrane protein crystals formed within the lipid cubic phase (LCP), where the LCP generates strong background scatter and is also difficult to remove from around the crystals 18 .

The new Versatile Macromolecular Crystallography microfocus (VMXm) beamline at Diamond Light Source provides the conditions with which to collect data from crystals potentially measuring less than a micron in size. The beamline has been designed to deliver a beam profile measuring 0.3 $\mu \mathrm{m} \times 0.5 \mu \mathrm{m}(\mathrm{VxH})^{1}$, a goniometer with a sphere of confusion no greater than $60 \mathrm{~nm}$ and an in vacuo sample environment. These design features of the VMXm endstation minimize the generation of background $\mathrm{X}$-ray noise by the beamline apparatus during data collection with the largest remaining source of background generated by the sample ${ }^{14}$.

Specific sample preparation methods designed for the VMXm beamline provide an opportunity to reduce this background and further improve the signal-to-noise of diffraction data, maximizing the quality of the data that can be recorded from microcrystals measuring $<10$ $\mu \mathrm{m}$. Many of the requirements outlined here for lowbackground diffraction from microcrystals are also common to cryogenic transmission electron microscopy (cryoTEM) ${ }^{19}$ and microcrystal electron diffraction (microED) ${ }^{20}$. As a result, many of the tools that have already been developed for the preparation of cryoTEM samples are suitable, with some adaptations, for the preparation of microcrystals. In the preparation of samples for single particle cryoTEM, the particles under investigation are embedded in very thin layers (typically $<100 \mathrm{~nm}$ ) of vitreous ice such that electrons are able to transmit through the sample. The thin uniform layer is achieved by blotting away excess liquid and vitrification of the sample is achieved by rapid cooling of the sample $(\sim 104$ $\left.\mathrm{K} \mathrm{s}^{-1}\right)^{21}$ through plunging into liquid ethane held at $\sim 93 \mathrm{~K}^{22}$. In contrast, liquid nitrogen, as used routinely for MX sample preparation, is a less efficient cryogen than ethane and has a greater propensity for crystalline ice formation within the sample ${ }^{21}$. The formation of crystalline ice, which can degrade diffraction and generate background noise, is normally mitigated through the use of cryo-protectant compounds ${ }^{23}$. Low molecular weight polymers such as poly-ethylene glycol (PEG) 400 and methyl-2,4-pentanediol (MPD), sugars, oils or saturated salts can be added to an aliquot of crystallization 
solution in low concentrations ${ }^{24}$ - there is not a 'one size fits all' solution to selecting the most appropriate cryoprotectant and this often requires optimization ${ }^{25}$. The crystal also undergoes multiple manipulations during the harvesting and cryo-protecting process which may result in damage to the crystal, the opportunity to utilize liquid ethane allows the omission of this step and helps protect the integrity of the crystal.

While liquid ethane is an effective cryogen for microcrystals $(<10 \mu \mathrm{m})$ due to the thinness of the sample, there are alternative methods for preventing crystalline ice formation, particularly in larger crystals, including reducing the water content of the crystal by use of a tightly controlled humid environment ${ }^{26}$, or through the wicking of excess liquid away from both the loop and surface of the crystal ${ }^{27}$, however, these again require greater manipulation of the sample. The use of automated blotting and plunge freezing with liquid ethane, as in cryoTEM, together remove excess crystallization solution and provide a means to flash cool microcrystals in a controlled manner while attempting to minimize manipulation.

Here, we present a protocol that can be utilized not only by both users of the VMXm beamline and at other microfocus beamlines to collect high signal-to-noise diffraction data but may also be useful to those preparing soluble protein crystal and detergent based membrane protein crystal samples for microED experiments. While all of the facilities to prepare and assess samples are available at VMXm, many structural biology laboratories are increasingly equipped for cryoTEM sample preparation. As a result, we envisage that some users may wish to use their own facilities to prepare their samples for beamtime at VMXm.

\section{Protocol}

\section{Equipment setup}

NOTE: The methods described here use a plunge freezing instrument with a single blotting arm. Some instruments are equipped with two blotting arms and we advise the user to check manufacturers' instructions to adjust the instrument so only one blotting arm is in use.

1. Ensure that a light microscope is positioned near to the plunge freezing instrument, ideally so that both the microscope and plunge freezer are in easy reach of the user.

2. Set up and cool the automated plunge freezer according to the manufacturers' instructions.

NOTE: The sample chamber temperature should be set to the temperature at which the crystals were grown. Do not place blotting paper in the sample chamber.

CAUTION: Liquid ethane is a highly flammable explosive and must only be used in a well-ventilated area away from potential spark sources.

3. Label grid boxes appropriately and cool them in a small dewar using liquid nitrogen.

4. Carefully place grids, carbon film side up, on an appropriate carrier for glow discharging (such as a glass microscope slide wrapped in parafilm).

5. Glow discharge cryoTEM grids for $25 \mathrm{~s}$ at $0.39 \mathrm{mBar}$, using a current of $15 \mathrm{~mA}$. Glow discharge just before the grids will be used. Keep the glow discharged grids in a covered Petri dish until ready; if $\mathbf{3 0}$ min lapses after glow discharge, repeat the glow discharging.

NOTE: With the plunge freezer ready and grids prepared, attention can turn to the sample. 


\section{Determining initial blotting parameters}

1. Set the relative humidity of the sample chamber to $90 \%$ and the blotting time to $5 \mathrm{~s}$ and ensure that the plunge freezer is set to automatically plunge the sample after blotting is complete.

NOTE: These starting parameters are most suitable for the Leica GP plunge freezer, other parameters such as blotting force are available on the FEI Vitrobot. However, in our experience crystal integrity is more likely to be maintained by the use of a single blotting arm.

2. To be able to seal the crystallization tray once the well of interest is opened, cut a small strip of tape and fold over one end to create a tab to ease opening of the seal and carefully place to one side.

3. Cut open the seal over the crystallization well, including the reservoir. Working quickly, apply $2-5 \mu \mathrm{L}$ of reservoir solution to the crystal containing drop to maintain liquid volume in the drop.

4. Use the tab of tape to then reseal the well and ensure that the crystallization drop does not dry out.

5. While momentarily holding open the tape over the crystallization well, transfer $10 \mu \mathrm{L}$ of the reservoir solution to a $0.5 \mathrm{~mL}$ tube to use for later steps.

6. Reseal the well.

7. Place a piece of pre-cut blotting paper on the blotting arm of the plunge freezing instrument.

8. Place a single glow discharged grid in the plunge freezing forceps and load into the instrument such that the carbon side faces away from the blotting arm.

9. Ensure that the relative humidity within the blotting chamber is at $90 \%$.
10. Rotate the forceps so the carbon-film side faces the blotting arm.

11. Using a $2.5 \mu \mathrm{L}$ pipette, apply $2 \mu \mathrm{L}$ of reservoir solution from the $0.5 \mathrm{~mL}$ tube to the non-support (shiny copper) side of the cryoTEM grid.

12. Rotate the grid so that the carbon support side is facing away from the blotting arm and repeat the process, carefully applying the liquid to the carbon-film support side of the grid. Avoid touching the carbon-film with the pipette tip so as to not damage the carbon-film. The liquid should spread across the grid due to the charge deposited during glow discharging.

13. Initiate the blotting process while observing the grid. A viewing scope is available on the Leica GP2 for this purpose.

14. Observe whether the liquid is drawn from the carbon surface of the grid during this time, this starts with the majority of the liquid bolus on the grid flattening out as the liquid is wicked through the grid. As the liquid in each grid square is reduced further, a wave of 'popping' across the surface of the grid can be observed. If this effect is observed, blotting can be stopped within 2-3 s of the popping effect ending. It is not necessary to plunge the test grid which can be discarded.

15. If the so-called 'popping' effect is not observed, repeat steps 2.11-2.14, each time extending the blotting time by 1-2 $s$ until the popping effect is observed just before the blotting arm retracts from the grid. Note this time for step 3.

NOTE: It is important that blotting stops as soon as this popping effect has occurred to ensure that during the process, crystals do not become dehydrated from overblotting. Use the crystallization solution from the reservoir 
for back blotting and dilution of the sample, as it ensures that the crystals are subject to a consistent solution, reducing the risk of destabilizing the crystals.

\section{Harvesting crystals}

1. Place the crystallization plate under the light microscope and position the target well within the field of view.

2. Place a fresh, glow discharged grid in the plunge freezer forceps and mount the forceps in the plunge freezer, with the carbon-film side facing away from the blotting arm.

3. Rotate the forceps so the carbon-film side faces the blotting arm.

4. Using a $2.5 \mu \mathrm{L}$ pipette, apply $2 \mu \mathrm{L}$ of reservoir solution from the $0.5 \mathrm{~mL}$ tube to the non-support side of the cryoTEM grid and rotate the grid so that the carbonfilm support side is facing the sample port of the plunge freezer.

5. Peel back the temporary seal and with the pipette set to 2 $\mu \mathrm{L}$, gently aspirate the crystallization drop repeatedly to suspend the microcrystals (it is important to not introduce air bubbles to the drop).

NOTE: It is critical to observe this process under the light microscope to ensure that crystals are released from any surface skin or the base of the well. If the crystals are stuck and not drawn up with aspiration, either the pipette tip or other crystallization tools such as an acupuncture needle, can be used to gently dislodge the crystals. Depending on the size of the crystals and the depth of field of the light microscope, it is sometimes possible to view, using the microscope, the crystals entering the pipette tip.
6. Transfer $2 \mu \mathrm{L}$ of the aspirated microcrystal slurry to the plunge freezer and apply all the sample to the carbon side of cryoTEM grid.

7. Blot for the time determined in step 2 and immediately initiate plunge-freezing. While blotting, observe for the occurrence of a popping wave effect across the grid and note whether this occurred completely across the grid. The presence of crystals can affect the initial blotting time, and this may need to be adjusted for subsequent grids by $1-2 \mathrm{~s}$.

8. Working quickly, transfer the grid from the liquid ethane to the grid box immersed in liquid nitrogen. Residual ethane can turn to an opaque white solid on the grid when it enters liquid nitrogen. To reduce this, remove the grid steadily from the liquid ethane, then quickly transfer to the liquid nitrogen reservoir.

9. Once 4 grids have been placed in the grid box, secure the lid of the box with the screw and transfer it either to a foam dewar of liquid nitrogen to allow for further sample assessment with a scanning electron microscope (SEM) or to a liquid nitrogen storage dewar using an appropriate storage container.

\section{Sample density assessment by light microscopy}

CAUTION: This method is destructive. If there is limited availability of sample, such as only one or two crystallization droplets, it is recommended that this step is skipped. After plunge freezing samples (step 3.7) the distribution of crystals across the grid can be assessed.

1. Mount a dry, room temperature cryoTEM grid in the plunge-freezer forceps and place the forceps on their side on the light microscope, with the grid in the field 
of view. Set an appropriate magnification and focus so that the whole grid and individual grid squares can be resolved.

2. Follow steps 3.1-3.7.

3. Instead of transferring the grid to the grid box (step 3.8), retract the plunged grid from the liquid ethane by resetting the plunge-freezer.

CAUTION: The grid and sample will warm to room temperature and cannot be used for further diffraction experiments.

4. Remove the forceps from the plunge freezing instrument.

5. Whilst holding the grid within the forceps, place the grid under the light microscope - the focus will already be roughly set.

6. Perform a fine focus and assess the density of the crystals across the grid. A good grid will contain several isolated crystals away from the grid bars within each grid square and clumping of crystals is minimal.

7. Where the density of crystals within the grid results in clumping of crystals with only a few isolated crystals, further dilute the crystal slurry with reservoir solution and repeat step 3. Take care when diluting samples so that the dilution does not result in the dissolution of the crystals.

8. Dilute the crystals in steps using small volumes of $0.5-1$ $\mu \mathrm{L}$.

\section{Sample assessment by scanning electron microscopy}

NOTE: Microcrystal preparation on cryoTEM grids is best assessed with a scanning electron microscopy (SEM) under cryogenic conditions, this can be achieved with an SEM fitted with a cryo-stage. At VMXm, a JEOL JSM-IT100
SEM (tungsten source) with a Quorum PP3006 airlock and cryostage are utilized. To ensure minimal radiation damage when viewing samples on $\mathrm{VMXm}{ }^{28,29}$, the following settings are used: $5 \mathrm{kV}$ accelerating voltage; a spot size of 40 (arbitrary units on the JEOL JSM-IT100); $10 \mathrm{~mm}$ working distance. Images are recorded using the secondary electron detector, for sample alignment and focusing a dwell time of $0.8 \mu \mathrm{s}$ is used while high-resolution images of single crystals are captured using a dwell time of $16 \mu \mathrm{s}$. It is important before loading a sample into the SEM that the microscope is aligned as per manufacturer instructions. It is advised that only a single grid is loaded into the SEM while any remaining grids prepared with the same parameters are reserved for X-ray diffraction experiments.

1. Prepare the SEM by cooling the sample cryo-stage to $-180{ }^{\circ} \mathrm{C}$ as per manufacturer's instruction. Follow the instructions for loading cryoTEM grids into the specific system available.

2. With the sample loaded in the SEM, turn on the electron beam, align sample and set focus using a high magnification (0.8 $\mu$ s dwell time).

3. First, perform the initial assessment with the whole of the grid in view at low magnification (x45). Record an image at this magnification.

4. Increase the magnification to allow closer inspection of individual grid squares, individual crystals should be very clearly observable.

5. Move around the grid, capturing still images (16 $\mu \mathrm{s}$ dwell time) of crystals ensuring they meet the following requirements before proceeding:

1. Ensure that grids are flat, and the carbon support film is largely intact. 
2. Ensure that there are numerous single crystals with a narrow halo of vitrified liquid surrounding the crystal.

3. Ensure that the holes in the carbon support film are visible.

4. Ensure that there are no large regions of vitrified liquid as defined by a dark and smooth appearance.

5. Ensure that there is little or no hexagonal ice, or surface ice scattered across the grid.

6. Ensure that crystals are evenly distributed across the support film and are not overlapping.

6. At this point, accurately measure the dimensions of a number of crystals to allow for an accurate X-ray beam size correlation during later diffraction experiments.

7. Samples that can be reliably retrieved from the SEM and maintained at cryogenic temperatures can be later used for X-ray diffraction experiments. If this is not possible, dispose of these samples.

NOTE: While imaging both the whole grid and individual microcrystals (approximately $\times 45$ and $\times 700$ magnification respectively), an assessment should be made on whether to proceed to the beamline with grids prepared using the same parameters or whether some parameters may need adjusting during step 3 . The grids should meet the tests described in step 5.5. If the grids do not meet these criteria, further optimization during step 3 is required before repeating step 5 .

\section{Preparing grid for diffraction experiments at VMXm}

1. Cool the required number of VMXm sample holders (Figure 4A) (up to 5) loaded into the sample cartridge (with lid) with the sample loader in a large foam dewar
(Figure 4B) using liquid nitrogen - this can require a large volume of liquid nitrogen. The liquid nitrogen level should be just above the sample position on the sample loader.

2. Prepare circlips and tools.

3. Place tools including clipping tool, forceps and VMXm sample forceps, into holes in the sample loader to cool. It can be useful to arrange a spotlight above the dewar.

4. Swiftly transfer the grid box containing the microcrystal loaded grids to the grid box recess on the sample loader and unscrew the lid such that it is loose and rotatable (do not remove).

5. Under liquid nitrogen remove the lid from the sample cartridge with large forceps and place it underneath the sample loader.

6. Use the VMXm sample forceps to lift the sample holder onto the sample loader, ensuring that the holder is facing upwards so that it can accept a grid. When in the correct position, a small pin on the sample loader will engage with the small hole in the middle of the sample holder.

7. With the cooled fine forceps, carefully lift the grid from the grid box and transfer close to the grid opening on the sample holder. Rotate the grid so that it lays flat (it does not matter which way the support film side is facing).

8. Gently lower the forceps so that the grid is as close as possible above the grid opening (be careful to not bend the grid) and release the grid into the opening. If the grid does not seat properly, carefully use the fine forceps to nudge the grid into position or gently tap the sample holder with the larger forceps.

9. Swiftly place the pre-cooled circlip tool over the grid in the grid opening and seat the circlip by pressing the button. 
It can be helpful to apply 2 depressions of the button to ensure the circlip is properly seated.

10. Top up the liquid nitrogen so the level is $\sim 1.5 \mathrm{~cm}$ above the sample holder. Using the VMXm sample forceps, carefully lift the loaded sample holder up and over the small pin on the sample loader and back into the sample cartridge. Note the position number of the sample holder in the sample cartridge.

11. Continue to load grids into the sample holders until all samples are loaded.

12. Return the grid boxes to the storage dewar if samples remain inside and remove the sample loader from the dewar to create more space.

13. Replace the cartridge lid on the cartridge, ensuring that the pin on the top of the cartridge engages with the hole in the lid.

14. Cool and fill the VMXm airlock dewar with liquid nitrogen and place next to the foam dewar containing the loaded sample cartridge.

15. Using the VMXm cartridge tool, carefully engage the tool in the ridges on the side of the cartridge and swiftly lift the cartridge into the airlock dewar.

16. Ensure that the liquid nitrogen level covers the cartridge.

17. Place lid on the airlock dewar and proceed to the VMXm endstation with the loaded sample cartridge.

NOTE: Loading of the sample cartridge into the VMXm endstation will be performed by beamline staff.

\section{Representative Results}

The aim of this protocol is to achieve vitrified microcrystals with a minimal volume of liquid surrounding the crystal that enable X-ray diffraction experiments with minimal background scatter to improve the diffraction signal. Example SEM images of microcrystals prepared on cryoTEM grids for minimal background scatter are shown in Figure 1A,B and

Figure 2. Grids with evenly distributed single crystals will provide the most efficient use of the grid, with good signalto-noise. However, this is not usually possible across the entirety of the grid and some regions may display some amount of clumping (Figure 2A,B). Despite this clumping, these examples still display a useful number of single isolated crystals that will provide low-background diffraction (Figure 5). The level of blotting that still maintains low-background scatter can vary. Strong blotting such that the holes in the carbon support film are clearly visible but the crystals remain hydrated is the aim (Figure 2C,D). However, good quality grids can still display some liquid within the holes of the support film although the position of the holes should still be identifiable (Figure 2A,B). Importantly, all of these examples display single, isolated crystals with a vitrified halo of liquid surrounding the crystal to maintain hydration between blotting and plunge freezing.

Many samples may require further optimization (Figure 3), which can include variation of the blotting time or the concentration of the microcrystals. Grids overloaded with crystals will demonstrate reduced blotting efficiency and can result in multiple lattices being recorded in single diffraction images (Figure 3A,B). More viscous crystallization conditions, such as that for trypsin which contains 8\% PEG 4000 and 15\% ethylene glycol (Figure 3C), can result in the need for longer blotting times (>10 s). Conversely, crystallization conditions with very low viscosity that blot very quickly, can suffer from distribution problems due to gravity causing settling before blotting occurs, resulting in all the microcrystals settling along one side of the grid (Figure 3D). 
Optimum preparation of samples allows the full capabilities of VMXm to be exploited to collect high quality X-ray diffraction data at the highest possible resolution with high signal-to-noise (Figure 5). These samples benefit from being compatible with the in-vacuo sample environment, resulting in very low or zero average background counts in diffraction images. The use of liquid ethane, without cryo-protectant, results in an absence of ice-rings (Figure 5), although where crystals lay close to the copper grid bars, the X-ray beam can glance the bars resulting in copper powder diffraction rings at $\sim 2.1 \AA$ and $\sim 1.8 \AA$.
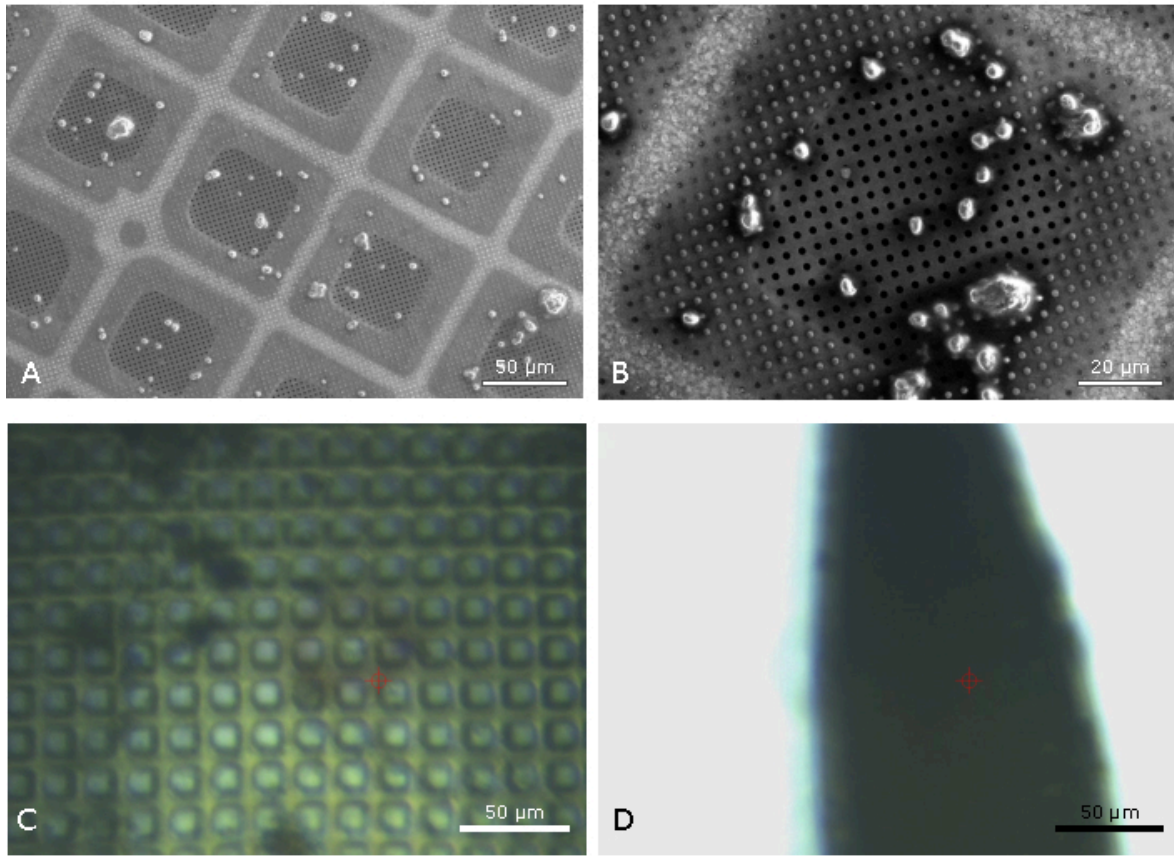

Figure 1: Comparison of microcrystal mounting on micromesh mounts and cyoTEM grids. Scanning electron micrographs of plunge frozen microcrystals of virus polyhedral measuring $2.5 \mu \mathrm{m}$ across $(\mathbf{A}, \mathbf{B}) 5 \mathrm{kV}$ accelerating voltage, a spot size of 39 (arbitrary units) and dwell time of $16 \mu \mathrm{s}$. The grid is free from excess liquid (A), a narrow halo of liquid is observed surrounding the crystals (B). The same samples mounted on a micromesh (20 $\mu \mathrm{m}$ apertures) before flash cooling in liquid nitrogen and observed using the on-axis-viewing system at beamline I24 face-on (C) and side on (D). The optical distortions (C) when viewing face-on give an indication of the extent of the liquid thickness across the micromesh, this is clearer when viewing the micromesh side-on (D). The red target in $\mathbf{C}$ and $\mathbf{D}$ represents the $\mathbf{X}$-ray beam position and size. Please click here to view a larger version of this figure. 

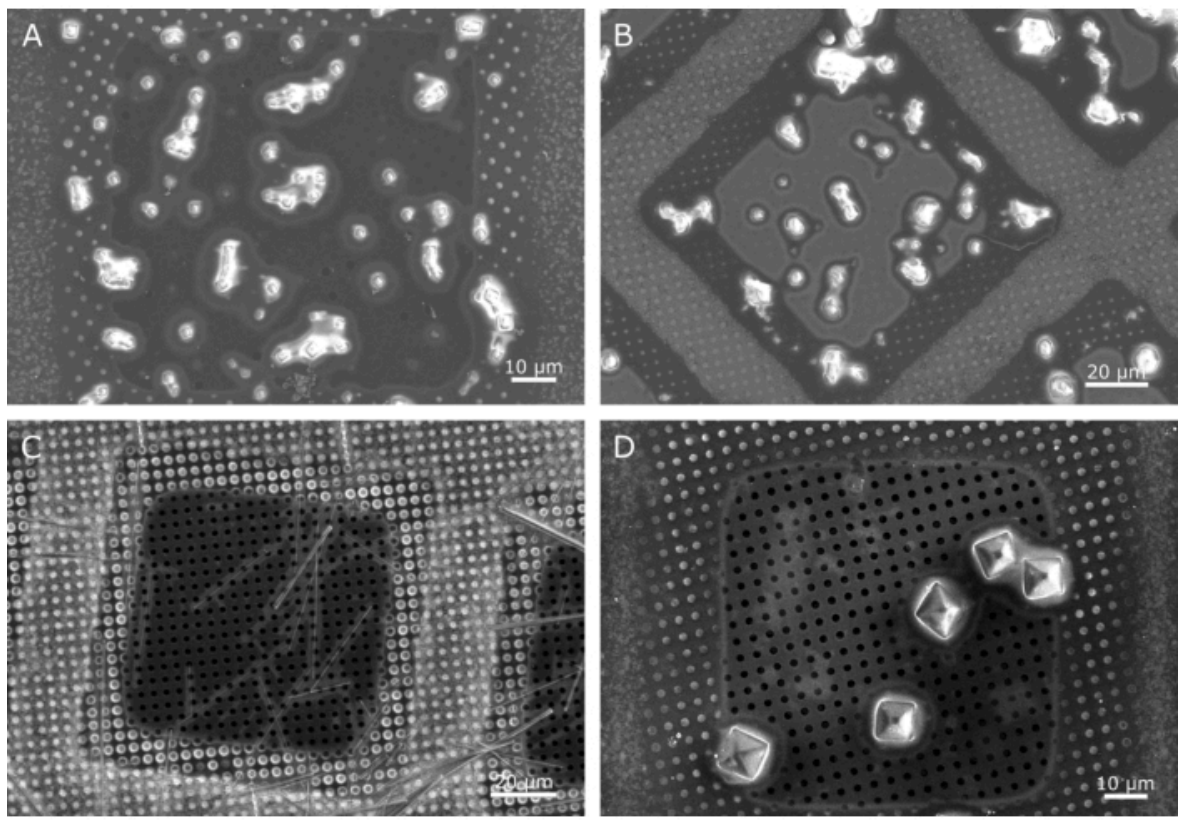

Figure 2: Examples of good quality samples. Polyhedra crystals (A) observed across a single $\sim 100 \mu \mathrm{m}$ grid square. While some of the crystals are slightly clumped and show some connectivity of the surrounding liquid, there are a number of isolated single crystals with a small halo of liquid surrounding the crystal. Slightly larger insulin crystals (B) measuring $\sim 5 \times 5 \mu \mathrm{m}$ also show some clumping but again there are well isolated individual crystals. Needle crystals can have a very narrow dimension and require a microbeam, such as these needle shaped lysozyme crystals (C). A narrow band of liquid can be seen surrounding these crystals (light grey halo). Larger microcrystals up to tens of microns also can be well mounted on cryoTEM grids, such as these $\sim 7 \mu \mathrm{m}$ proteinase $\mathrm{K}$ crystals (D). In both (C) and (D) and to a lesser extent in (A), the holes of the carbon support film are clearly visible, demonstrating the presence of very little/no liquid. In example B, while no empty holes are observed, the position of the holes can still be identified, indicating that the liquid is only filling the holes in the support film. In all of these examples, a halo of liquid can be seen around the edge of the grid square (a rounded inner square), where the holes have a lighter grey appearance. This is a common feature of well-prepared grids. Please click here to view a larger version of this figure. 

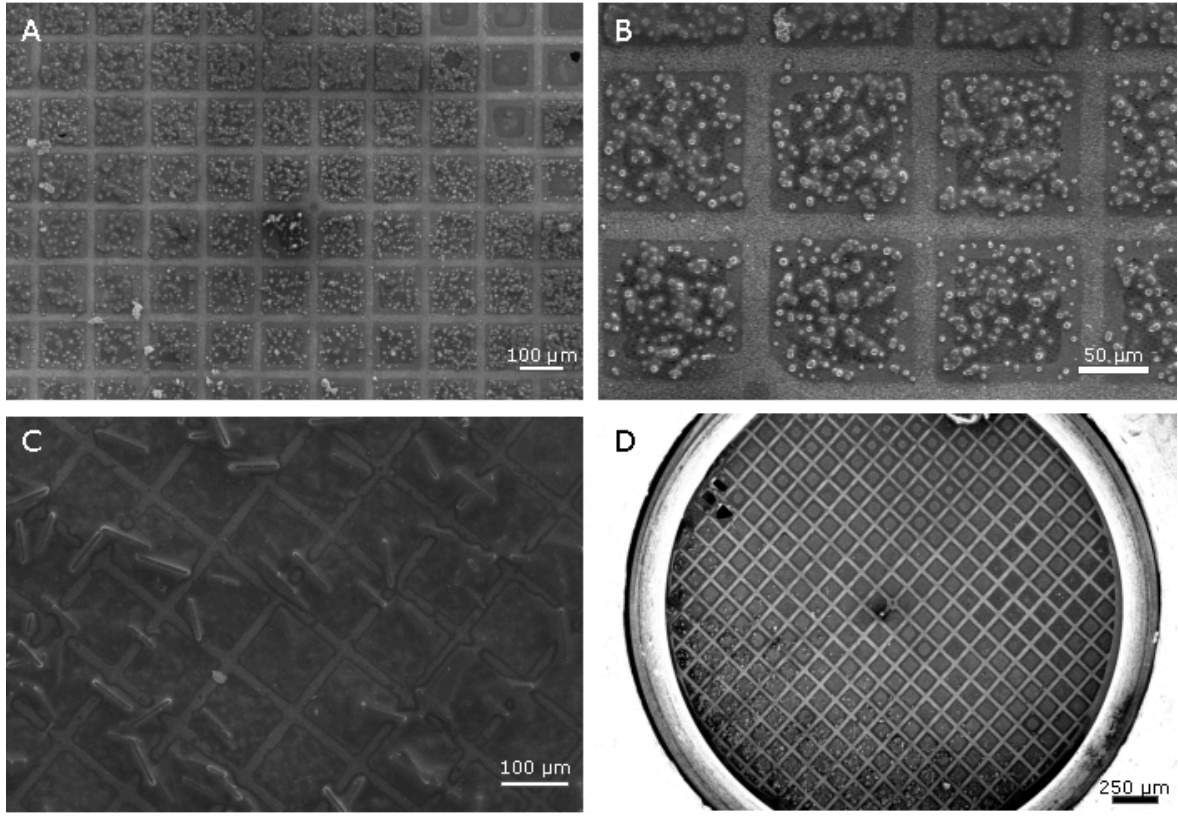

Figure 3: Examples of samples requiring further optimisation. Grids overloaded with microcrystals (A, B) can increase the background scatter as the samples block the holes in the support film reducing the blotting efficiency, and with a higher surface tension between the crystals more liquid remains. As well as a degradation in the signal-to-noise resulting in a loss of information, it is likely that multiple lattices will be recorded. Using a blotting time that is not long enough, or a highly viscous crystallization solution can result in an overly wet sample (C), also producing low signal-to-noise. Crystallization conditions with no precipitants, such as those for bovine insulin (D), have a very low viscosity. While this results in a very short blotting time, it can also result in the movement of crystals across the grid before and during blotting due to the effects of gravity. This usually results in a largely empty grid with a high concentration of crystals along one edge (D). It can be advantageous to add a viscous agent such as ethylene glycol to the crystal slurry shortly before applying them to the grid to reduce crystal flow and improve the distribution of the crystals. This can also lengthen the blotting time, making it easier to observe blotting. Please click here to view a larger version of this figure. 

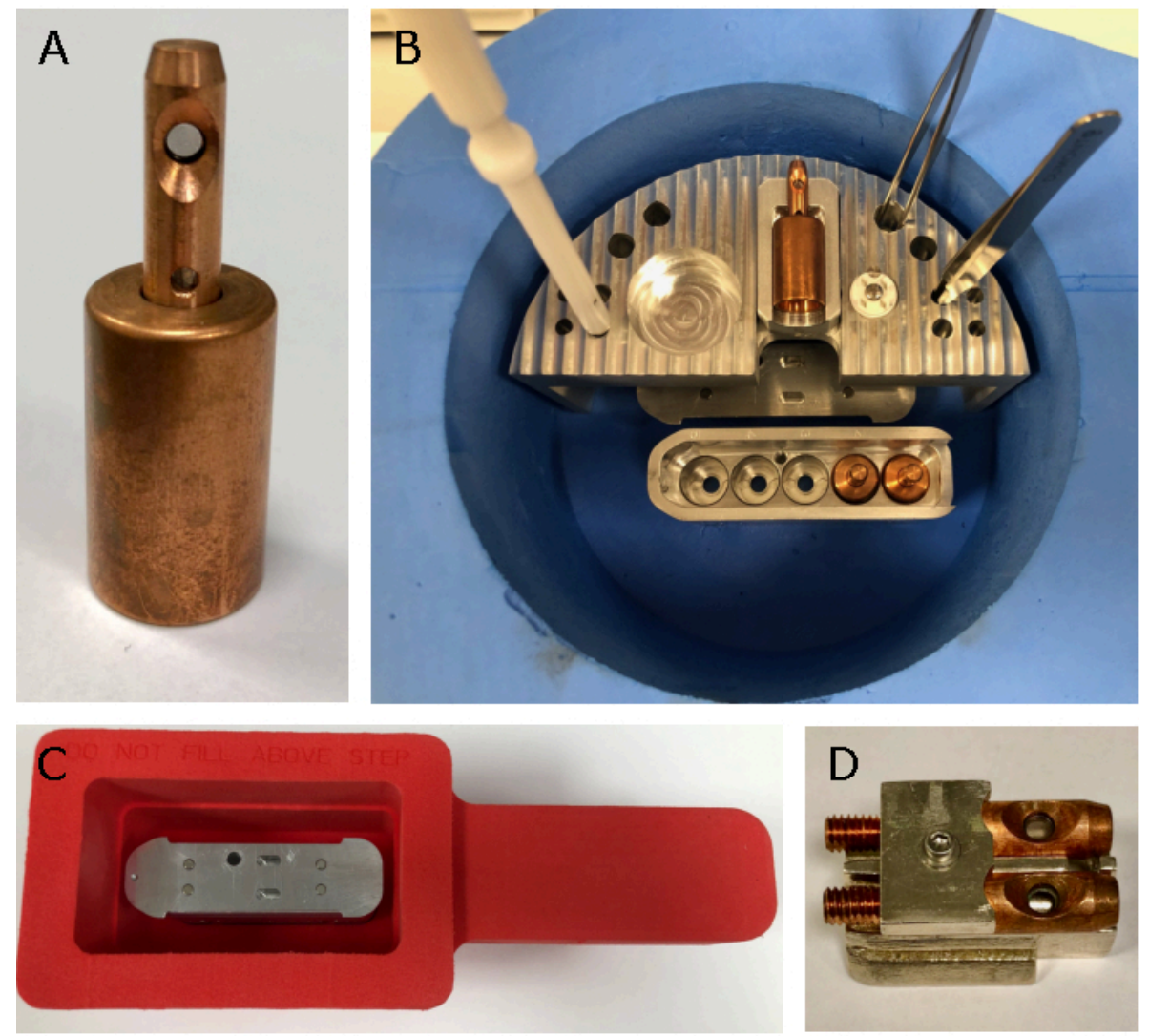

Figure 4: Tools for sample loading at VMXm. A bespoke set of tools has been designed to load the VMXm sample holders (A). The sample loader (B) has space for a single VMXm sample holder, grid box and tool storage while working. It is also designed to allow working just below the surface of the liquid nitrogen and allow the sample cartridge to fit beneath (B). The airlock dewar (C), which fits the airlock nitrogen gas box on the VMXm endstation, is used to take the loaded sample cartridge from the lab to the beamline experimental hutch. To view samples in the offline SEM, a bespoke shuttle (D) comprising of the VMXm sample holder without base has been made to enable assessment in the sample holder that is used on the beamline. Please click here to view a larger version of this figure. 

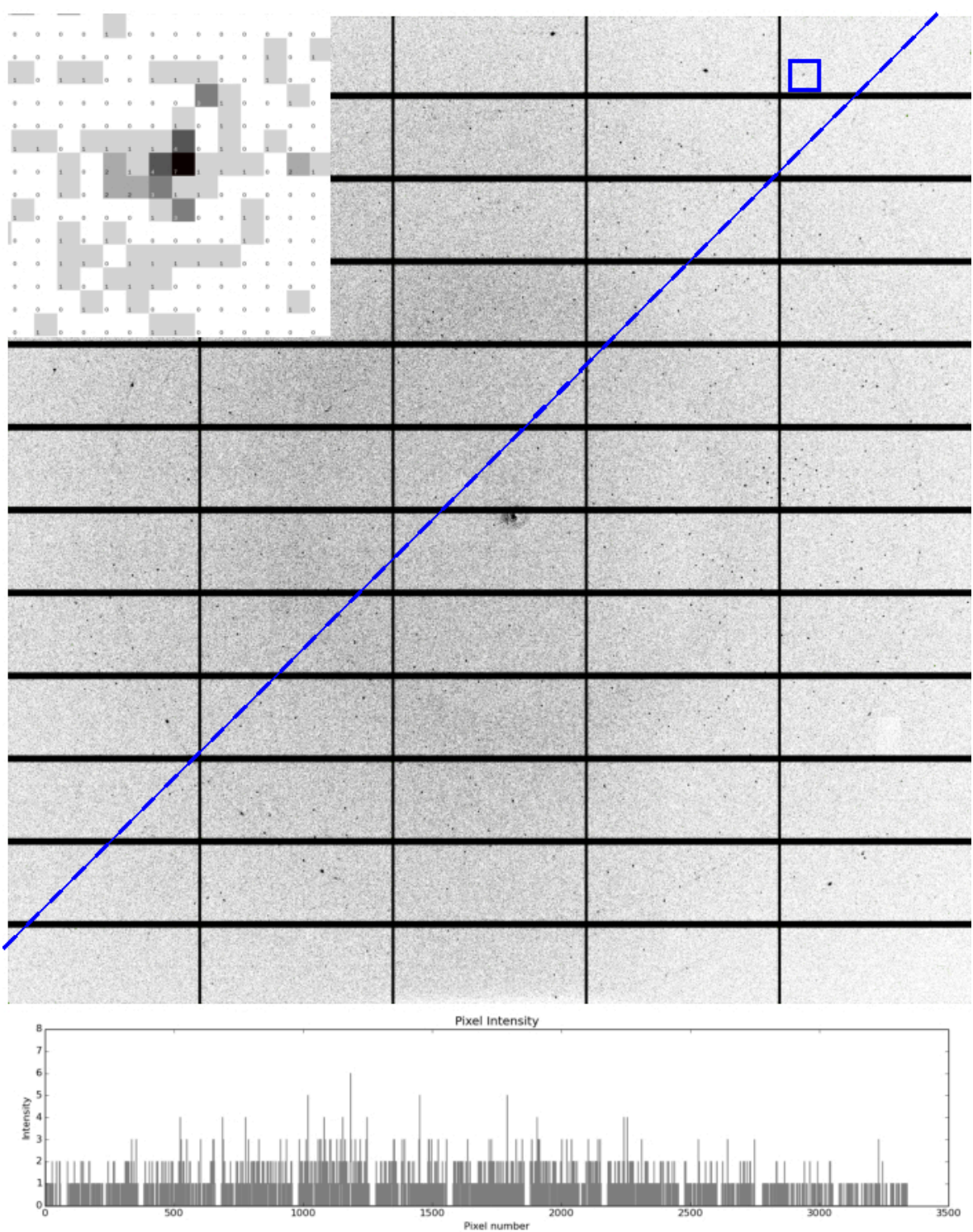

Figure 5: Example diffraction from microcrystals at VMXm. A single diffraction image recorded using a Pilatus $36 \mathrm{M}$ detector at VMXm of a $\sim 3 \mu \mathrm{m}$ virus polyhedral crystal mounted on a cryoTEM grid using the plunge freezing method. Diffraction is observed to beyond $1.7 \AA$ and a reflection (blue square) at $1.74 \AA$ is inset, demonstrating the low background scatter, with a high number of pixels with zero counts. Ethylene glycol to a final concentration of $50 \% \mathrm{v} / \mathrm{v}$ was added to the crystal slurry before applying it to the grid. The low background nature of the VMXm sample position is demonstrated by the constant background across the image as shown by intensities plotted beneath the blue dashed line, even near the beam center. The plotted intensities show the background remains below 3 counts. Two faint rings are observed at $2.15 \AA$ and $1.86 \AA$ which are generated by powder diffraction of the copper of the cryoTEM grid. There are no detectable ice-rings, 
demonstrating the effectiveness of blotting followed by cooling with liquid ethane. Please click here to view a larger version of this figure.

\section{Discussion}

This protocol demonstrates how tools from cryoTEM sample preparation can be used for the preparation of microcrystals for X-ray diffraction experiments at microfocus beamlines. Standard beamline instrumentation is centered around a pin mounted sample and while efforts have been made to provide a sample support on these mounts for microcrystals, they are often challenging to load with sample whilst ensuring that the highest signal-to-noise is achieved (Figure 1C,D). Many of these samples may also require optimization of the cryo-protection conditions to ensure the sample is vitreous. The plunge freezing method provides a repeatable manner to remove excess liquid and flash cool the sample in an efficient cryogen (Figure 1A,B). While the grid can then be mounted on a standard beamline with a tweezer-based pin mount, VMXm sample holders have been specifically designed to accept grids and hold them below the glass transition temperature in a vacuum environment via conductive cooling. The sample environment of VMXm enables low background data collection, where the sample is the remaining source of background, and provides a microbeam that can be used to match crystals with dimensions less than $10 \mu \mathrm{m}$. This sample preparation method can also be used to prepare nanocrystals for electron diffraction where there is also a requirement for very little excess liquid and a vitreous sample due to the weak penetration of electrons. While cryoTEM grids are fragile, those experienced in the harvesting of crystals in loops will quickly adapt to the handling of the grids. With a small amount of experience, few grids will be lost during the blotting, freezing and loading stages of the protocol. The optimization steps are, however, critical to this success and careful preparation will reduce the chances of losing crystals or reducing crystal integrity.

CryoTEM grids provide a relatively large single mount that can contain many hundreds of crystals, thus improving throughput where it may only be possible to record a small wedge of diffraction data. A single grid may also provide enough crystals to determine the protein structure, particularly in crystals of high symmetry. Where only one or two single crystallization drops have generated microcrystals, trial blotting of the crystallization condition alone can help ensure that when the microcrystals are blotted, the times used are as close as possible to those needed to generate initial good quality samples. The carbon-film supports are invisible to X-rays and are available with differing hole spacing, which can be used to suit a particular morphology. We most commonly utilize support films with $2 \mu \mathrm{m}$ holes at a $2 \mu \mathrm{m}$ spacing, but smaller holes with greater spacing may be more appropriate for crystals smaller than $2 \mu \mathrm{m}$. Other support films such as those with $1 \mu \mathrm{m}$ holes with a $4 \mu \mathrm{m}$ spacing as well as support films with different shaped holes are available, all of which will affect the blotting time. A grid square mesh size of 200 (200 squares per inch) also provides enough space $(\sim 100 \mu \mathrm{m})$ between the copper grid bars so that the X-ray beam does not strongly interact with the copper while providing enough structural support for the carbon-film loaded with crystals. The use of liquid ethane negates the need for cryoprotectants, which in turn reduces the requirement on sample volume that would have been used in the optimization of cryoprotectant conditions.

The main parameters to be optimized during the process are the blotting times and the sample dilution. Blotting 
times should be long enough to observe the 'popping' effect across the entirety of the grid before plunge freezing. Over blotting could result in the dehydration of the crystals, however, control of the humidity within the sample chamber is used to minimize this effect. While it is suggested that a relative humidity of $90 \%$ used, some samples may benefit in optimization of the humidity. The humidity may affect the blotting efficiency of the blotting paper which can slowly become saturated with water. Additionally, humidity control within the sample chamber could be used to improve diffraction quality of the crystals ${ }^{30}$. It is recommended that small changes $(<5 \%)$ in humidity are made before checking diffraction integrity to ensure the diffraction quality is not degraded.

Optimization of non-precious samples can be conducted using a light microscope in place of an SEM. Although destructive, it is useful for assessing the density of crystals across the grid and to enable decision making on whether a sample should be diluted or concentrated to better disperse crystals across the grid. This step is most useful when there are a large number of crystals available and particularly highly concentrated samples. Clumping together of crystals should be avoided (Figure 3), as while it is not a significant problem if two crystals are illuminated at the same time during data collection ${ }^{6}$, there will likely be a larger volume of liquid surrounding the clump, thus reducing the signalto-noise (Figure 5). While it is possible to observe large excesses of liquid globally across the grid using a light microscope, assessment of the volume of liquid surrounding the microcrystals and the presence of crystalline ice can only be made using an electron microscope fitted with a cryogenic vacuum transfer system and stage. Sometimes, after application of the crystals to the grid and before blotting occurs, crystals in low viscosity solutions may settle along one edge of the grid. We have found that adding up to a $50 \%$ final concentration of ethylene glycol can slow the movement of crystals through the droplet, ensuring a better distribution of microcrystals across the grid as well as providing greater control over blotting by increasing the blotting time (Figure 3D).

Some crystallization solutions containing viscous precipitating agents such as high molecular weight PEGs can prove challenging to blot, requiring increasingly long blotting times (>10 s). In such cases, it can be helpful to reduce the volume of liquid deposited on the back of the grid as well as the volume of the crystal containing solution to the supportfilm side of the grid. Strategies such as using 2 layers of blotting paper or glass fibers may also aid blotting in these difficult cases ${ }^{31}$.

While this pipeline is suited to soluble protein crystals, those that form in very viscous mediums such as membrane proteins in LCP present a different challenge for which this protocol is not suited. However, strategies are emerging for preparing LCP crystals on cryoTEM grids for microED which include reducing the viscosity of the samples by inducing a phase change to the LCP. This permits the samples to be applied to grids in a similar manner to that described in this article. Finally, the sample can be milled with a focused ion beam to remove excess non-crystal material ${ }^{32,33,34}$.

Overall, this pipeline will generally take $1-2 \mathrm{~h}$ (including equipment setup time) to follow from the sample arriving at VMXm to providing optimized grids with well dispersed, vitrified samples, depending upon sample availability, the concentration of crystals and the viscosity of the crystallization solution. These methods have already been successfully employed for the preparation of microcrystals for $X$-ray diffraction experiments exploring radiation damage on 
microcrystals where a minimal volume of liquid surrounding the sample was essential 28,35 . It should be noted that the protocol can be applied to all soluble microcrystal samples, not just to well diffracting samples that have already been optimized. A crystallization experiment that produces microcrystalline material would traditionally be a target for optimization with the aim of obtaining larger crystals, however, this sample preparation method and the capabilities of VMXm may allow adequate data to be collected from such samples without further optimization. Alternatively, if such microcrystalline samples diffract poorly, the data collected from VMXm using this sample preparation method could still act as a useful guide for further optimization of crystallization conditions. The tools for preparing grids, including glow discharging and plunge freezing are now widely available in research institutes equipped for cryoTEM experiments and will be available to many users enabling them to prepare samples in advance of beamtime at VMXm.

\section{Disclosures}

No conflicts of interest to declare.

\section{Acknowledgments}

The authors would like to thank Jeremy Keown, Jon Grimes, Geoff Sutton and Dave Stuart, STRUBI University of Oxford and Rachel Bolton, University of Southampton for kindly providing microcrystal samples for the development and demonstration of sample preparation methods for the VMXm beamline in addition to enabling commissioning of the beamline. The authors would also like to thank iNEXTDiscovery (project number 871037 ) for the opportunity and support in publishing this manuscript.
1. Laundy, D. et al. Development of a multi-lane X-ray mirror providing variable beam sizes. Review of Scientific Instruments. 87 (5), 051802-051806 (2016).

2. McPherson, A., Gavira, J. A. Introduction to protein crystallization. Acta crystallographica. Section F, Structural biology communications. F70 (Pt 1), 2-20 (2014).

3. McPherson, A., Cudney, B. Optimization of crystallization conditions for biological macromolecules. Acta crystallographica. Section F, Structural biology communications. F70 (11), 1445-1467 (2014).

4. Holton, J. M., Frankel, K. A. The minimum crystal size needed for a complete diffraction data set. Acta Crystallographica Section D. D66 (Pt 4), 393-408 (2010).

5. Garman, E. F., Owen, R. L. Cryocooling and radiation damage in macromolecular crystallography. Acta Crystallographica Section D. D62 (1), 32-47 (2005).

6. Gildea, R. J. et al. New methods for indexing multi-lattice diffraction data. Acta Crystallographica Section D. D70 (Pt 10), 2652-2666 (2014).

7. Chapman, H. N. X-Ray Free-Electron Lasers for the Structure and Dynamics of Macromolecules. Annual Review of Biochemistry. 88 (1), 35-58 (2019).

8. Beale, J. H. et al. Successful sample preparation for serial crystallography experiments. Journal of Applied Crystallography. 52 1385-1396 (2019).

9. Smith, J. L., Fischetti, R. F., Yamamoto, M. Microcrystallography comes of age. Current Opinion in Structural Biology. 22 (5), 602-612 (2012).

10. Owen, R. L., Juanhuix, J., Fuchs, M. Current advances in synchrotron radiation instrumentation for MX

\section{References}


experiments. Archives of Biochemistry and Biophysics. 602 21-31 (2016).

11. Miller, M. et al. Getting the Most Out of Your Crystals: Data Collection at the New High-Flux, Microfocus MX Beamlines at NSLS-II. Molecules. 24 (3), 496-416 (2019).

12. Axford, D., Ji, X., Stuart, D. I., Sutton, G. In cellulo structure determination of a novel cypovirus polyhedrin. Acta Crystallographica Section D. D70 (Pt 5), 1435-1441 (2014).

13. Ji, X. et al. Polyhedra structures and the evolution of the insect viruses. Journal of structural biology. 192 (1), 88-99 (2015).

14. Evans, G., Axford, D., Owen, R. L. The design of macromolecular crystallography diffraction experiments. Acta Crystallographica Section D. D67 (Pt 4), 261-270 (2011).

15. MiTeGen. $<$ https://www.mitegen.com/product/ micromeshes/> (2021).

16. Guo, G. et al. Sample manipulation and data assembly for robust microcrystal synchrotron crystallography. IUCrJ. M5 238-246 (2018).

17. Cipriani, F. et al. Automation of sample mounting for macromolecular crystallography. Acta Crystallographica Section D. D62 (10), 1251-1259 (2006).

18. Caffrey, M. A comprehensive review of the lipid cubic phase or in mesomethod for crystallizing membrane and soluble proteins and complexes. Acta crystallographica. Section F, Structural biology communications. F71 (1), 3-18 (2015).

19. Thompson, R. F., Walker, M., Siebert, C. A., Muench, S. P., Ranson, N. A. An introduction to sample preparation and imaging by cryo-electron microscopy for structural biology. Methods. 100 (C), 3-15 (2016).

20. Nannenga, B. L. MicroED methodology and development. Structural Dynamics. 1-8 (2020).

21. Dubochet, J. et al. Cryo-electron microscopy of vitrified specimens. Quarterly Reviews of Biophysics. 21 (2), 129-228 (1988).

22. Tivol, W. F., Briegel, A., Jensen, G. J. An improved cryogen for plunge freezing. Microscopy and Microanalysis. 14 (5), 375-379 (2008).

23. Garman, E. F., Schneider, T. R. Macromolecular Cryocrystallography. Journal of Applied Crystallography. 30 (3), 211-237 (1997).

24. Pflugrath, J. W. Practical macromolecular cryocrystallography. Acta Crystallographica Section F. F71 (6), 622-642 (2015).

25. Alcorn, T., Juers, D. H. Progress in rational methods of cryoprotection in macromolecular crystallography. Acta crystallographica Section D. D66 (Pt 4), 366-373 (2010).

26. Bowler, M. W., Montgomery, M. G., Leslie, A. G. W., Walker, J. E. Reproducible improvements in order and diffraction limit of crystals of bovine mitochondrial $F(1)$-ATPase by controlled dehydration. Acta Crystallographica Section D. D62 (Pt 9), 991-995 (2006).

27. Pellegrini, E., Piano, D., Bowler, M. W. Direct cryocooling of naked crystals: are cryoprotection agents always necessary? Acta Crystallographica Section D. D67 (Pt 10), 902-906 (2011).

28. Beale, E. V. et al. Scanning electron microscopy as a method for sample visualization in protein X-ray crystallography. IUCrJ. 1-9 (2020). 
29. Hattne, J. et al. Analysis of Global and Site-Specific Radiation Damage in Cryo-EM. Structure. 26 (5), 759-766.e754 (2018).

30. Sanchez-Weatherby, J. et al. Improving diffraction by humidity control: a novel device compatible with X-ray beamlines. Acta crystallographica Section D. D65 (12), 1237-1246 (2009).

31. Tan, Y. Z., Rubinstein, J. L. Through-grid wicking enables high-speed cryoEM specimen preparation. Acta Crystallographica Section D. 76 (11), 1092-1103 (2020).

32. Martynowycz, M. W., Khan, F., Hattne, J., Abramson, J., Gonen, T. MicroED structure of lipid-embedded mammalian mitochondrial voltage-dependent anion channel. Proceedings of the National Academy of Sciences. 117 (51), 32380-32385 (2020).

33. Zhu, L. et al. Structure Determination from Lipidic Cubic Phase Embedded Microcrystals by MicroED. Structure. 28 (10), 1149-1159.e1144 (2020).

34. Polovinkin, V. et al. Demonstration of electron diffraction from membrane protein crystals grown in a lipidic mesophase after lamella preparation by focused ion beam milling at cryogenic temperatures. Journal of Applied Crystallography. 53 (6), 1416-1424 (2020).

35. Storm, S. L. S. et al. Measuring energy-dependent photoelectron escape in microcrystals. IUCrJ. 7 (1), 129-135 (2020). 